\title{
Successful combined surgical approach in a rare case of retrotracheal goitre in a patient with anatomical impediments
}

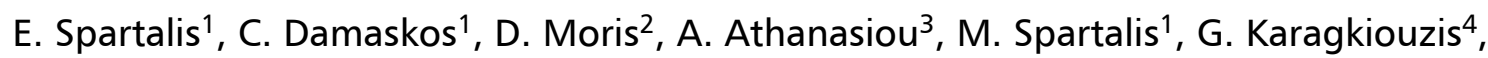 \\ D. Schizas ${ }^{5}$, D. Dimitroulis ${ }^{1}$, P. Tomos ${ }^{4}$ \\ $12^{\text {nd }}$ Department of Propaedeutic Surgery, University of Athens Medical School, Athens, Greece \\ ${ }^{2}$ Department of Surgery, The Ohio State University Comprehensive Cancer Centre, the Ohio State University, \\ Columbus, $\mathrm{OH}$, United States \\ ${ }^{3}$ Department of Surgery, Mercy University Hospital, Cork, Ireland \\ ${ }^{4}$ Department of Thoracic Surgery, University of Athens Medical School, Athens, Greece \\ ${ }^{5} 1^{\text {st }}$ Department of Surgery, University of Athens Medical School, Athens, Greece \\ [Received: 23 April 2017; Accepted: 27 July 2017]
}

\begin{abstract}
Diving goitres can descend the cervical region expanding directly into the thoracic cavity. In most cases, diving goitres extend into the anterosuperior compartment, but they may also extend behind the trachea. We herein present a case of a male patient with retrotracheal goitre and history of left thyroid lobectomy and median sternotomy for thoracic aortic aneurysm repair with graft placement. After detailed preoperative evaluation, the patient underwent surgical resection of the mass through a combined approach; the existing cervical incision and a right posterolateral mini-thoracotomy. The postoperative course of the patient was uncomplicated. One year after surgery, the patient is asymptomatic and disease-free. (Folia Morphol 2018; 77, 1: 166-169)
\end{abstract}

Key words: retrotracheal, goitre, aortic grafting, thyroidectomy, thoracotomy

\section{INTRODUCTION}

Diving goitres can descend the cervical region expanding directly into the thoracic cavity. In most cases, diving goitres extend into the anterosuperior compartment, but they may also extend behind the trachea [2]. These goitres tend to exhibit increased vascularisation arising from the lower thyroid poles. However, there is a possibility up to $1 \%$ for primary goitres emerging from ectopic thyroid tissue located in the anterior, the medium or the posterior mediastinum $[2,14]$. In this case, the blood supply does not originate from the inferior thyroid vessels. Exceptions also include recurrent goitres in which a substernal remnant from the initial surgery underwent neovascularisation based on parasitising mediastinal vessels [14].

\section{CASE REPORT}

A 68-year-old Caucasian male was presented to the surgical department complaining of mediocre difficulty swallowing solid food, dyspnoea and hoarseness; all symptoms have appeared recently. The physical examination is normal and the neck is supple without thyromegaly. His medical history included left thyroid lobectomy several years ago, median sternotomy for thoracic aortic aneurysm repair using a synthetic graft substernally and long

Address for correspondence: E. Spartalis, MD, MSc, PhD, $2^{\text {nd }}$ Department of Propaedeutic Surgery, University of Athens Medical School, Vasilissis Sofias 49, Athens 106 76, Greece, tel: +306974714078, fax: +302106416015, e-mail: eleftherios.spartalis@gmail.com 


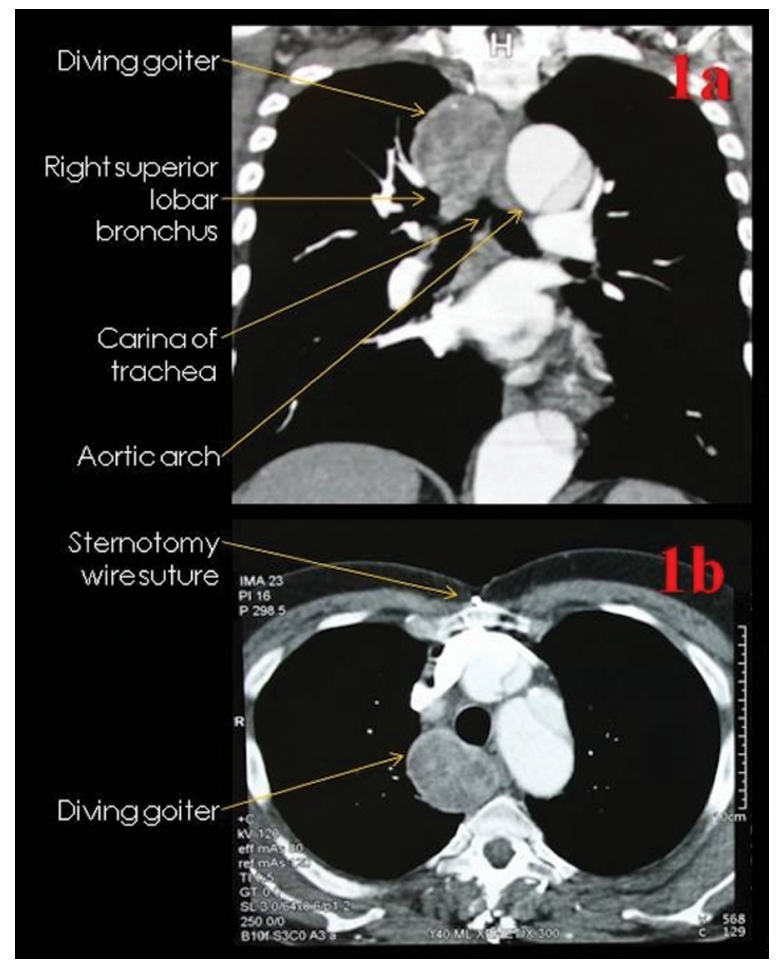

Figure 1. A. Contrast-enhanced coronal computed tomography scan image demonstrates a globulous, sharply demarcated lesion to the right of the trachea and oesophagus and airway compromised at the carina level. The lesion was inhomogeneous extending up to the vertebral body; $\mathbf{B}$. The oesophagus appeared to be remarkably compressed while the trachea was slightly displaced to the left.

term chronic medical treatment with amiodarone. On chest $\mathrm{X}$-ray, the anterior mediastinum is enlarged and the trachea is displaced to the left. Wires are placed upon the median sternal line. The cardiac silhouette is normal. The lung fields are clear and there are no pleural effusions.

Contrast-enhanced computed tomography (CT) scan images demonstrate a globulous, sharply demarcated lesion to the right of the trachea and oesophagus. The lesion is inhomogeneous, extending up to the vertebral body. The oesophagus appears to be remarkably compressed while the trachea is slightly displaced to the left (Fig. 1B). The coronal CT image shows airway compromise at the carina level (Fig. 1A).

After detailed preoperative evaluation, the patient underwent surgical thyroid gland resection through a combined approach; the existing cervical incision and a right posterolateral mini-thoracotomy. This was obligatory due to the acquired anatomic alterations caused by previous surgical interventions, as the anterior mediastinal architecture was affected by the

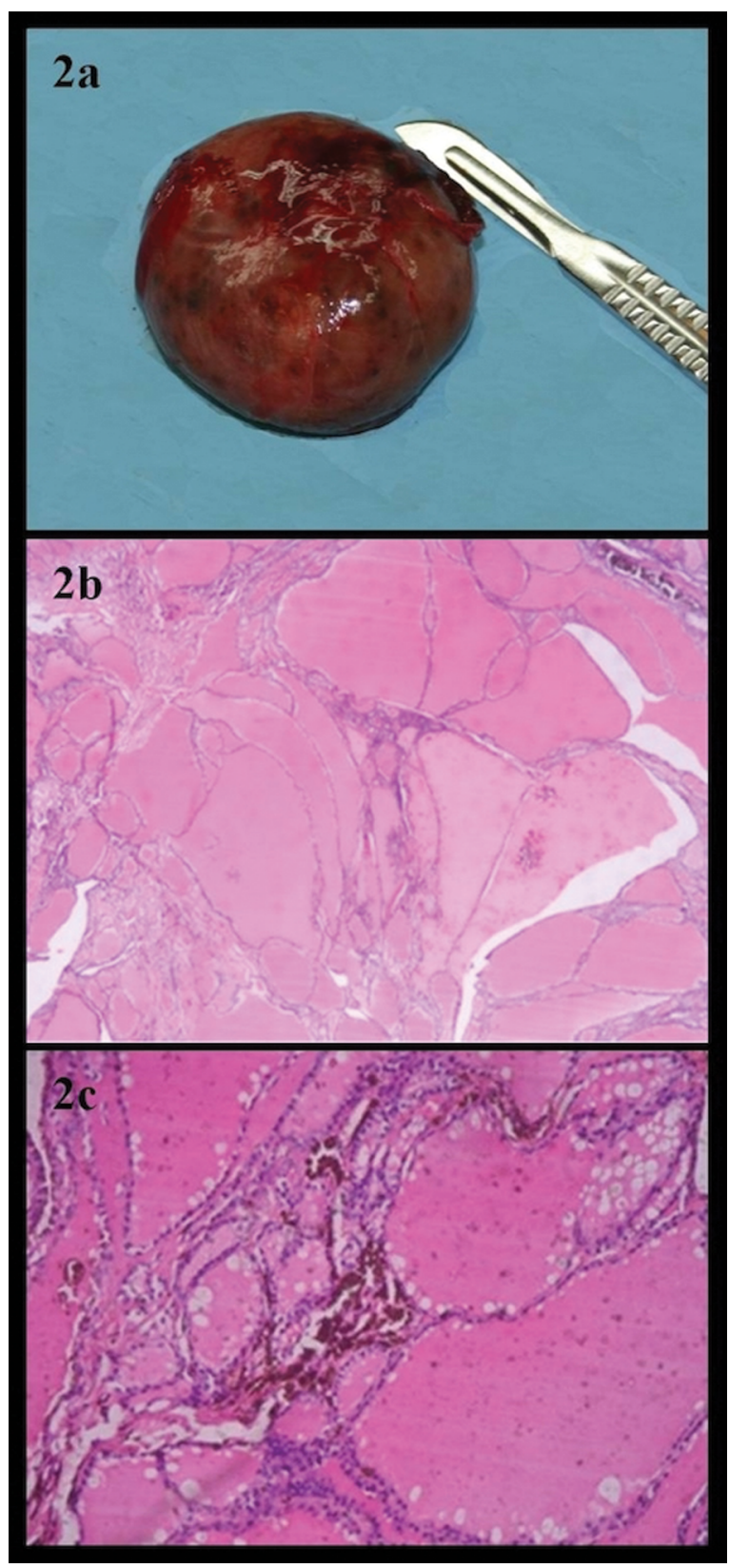

Figure 2. A. The resected intrathoracic specimen (size $6 \times 5.5 \times$ $\times 4 \mathrm{~cm}$ ); B. Histopathology of the lesion revealed multiple large pieces of thyroid tissue with thyroid follicles of varying size and shape, lined by uniform cuboidal cells; $\mathbf{C}$. The cells were filled with colloid with some follicles showing cystic macrophages in the lumen.

presence of the synthetic graft and post-operative fibrotic tissue.

The histopathological findings (Fig. 2) are compatible with thyroid gland tissue, appearing regions of colloid, nodular hyperplasia, cystic-fibrous degeneration and calcification present both at the two specimens sent for control. 
The postoperative course of the patient was uncomplicated. One year after surgery, the patient is asymptomatic and disease-free.

\section{DISCUSSION}

High suspicion of an intrathoracic goitre could be based on the trachea deviation appearing on a plain chest X-ray [2]. Chest X-rays and CT scanning are usually diagnostic, and additional evaluation is rarely necessary $[14,15]$. Initial laboratory workup should include thyroid function tests [15]. I-131 uptake scanning can help in order to certify the thyroid gland origin of the tissue in case we set as possible a lymphoma, a thymoma or a germ cell tumour diagnosis [17]. Needle biopsy is not necessary before resection [17]. These tumours should always be resected, even if asymptomatic, because of potential airway compromise, but it is crucial to obtain accurate preoperative imaging in order to plan the procedure accordingly [17].

The majority of the intrathoracic goitres can be safely excised through a standard cervical incision $[10,17]$. At this point, it is necessary to mention that sternotomy (partial or complete) often offers a better surgical field and is usually preferred if there is a large substernal component, if the tumour's blood supply arises from an intrathoracic source, or if the tumour appears to infiltrate surrounding structures $[5,10,17]$. A combined right thoracotomy and cervical approach can also be performed [5, 6]. Ectopic intrathoracic goitres (no cervical component) and substernal goitres in patients who have previously undergone cervical thyroidectomy should be approached by sternotomy or thoracotomy alone $[10,17]$.

Most mediastinal goitres are retrosternally situated in the anterior mediastinal compartment $[5,6$, 16]. Posterior mediastinal goitres, either retrotracheal or retroesophageal, are rare. Large posterior mediastinal, contralateral retrotracheal or retroesophageal goitres are best removed through a combined cervical and thoracic approach $[6,16]$. It has been reported that skilled head and neck surgeons, with adequate thyroid surgery experience, need to perform an extracervical approach in $2-5 \%$ of thyroidectomies for retrotracheal goitres, but some authors have reported an incidence of sternotomy in $29 \%$ of patients $[5,6$, $10,13,16]$.

This variability could be correlated with the lack of uniformity in the definition of a retrotracheal goitre $[6,8,9,11-13,16]$. Initially, a goitre was generically considered as retrosternal when extended below the thoracic inlet. Later, retrotracheal goitre was defined by De Souza and Smith [8] as a goitre with a portion of its mass $\geq 50 \%$ situated in the mediastinum. However, this definition lacks anatomic precision and can be interpreted unspecifically. More precise definitions of retrotracheal goitres have been suggested; namely, a goitre lying two fingerbreadths below the thoracic inlet with the patient in a supine position, a goitre reaching the aortic arch, or the carina tracheae, a goitre with its inferior pole passing through the cervicothoracic isthmus below the subclavian vessels. Several classification systems have also been developed in order to classify retrotracheal goitres better. Cohen and Cho [3] classify goitres into four grades, depending on the percentage of goitre mass located in the mediastinum. Huins et al. [11] proposed a classification of retrotracheal goitres based on the relationship of goitre with anatomical structures of the mediastinum: they defined three grades of goitre depending upon mediastinal extension, namely, to the level of the aortic arch, to the level of the pericardium or below the level of the right atrium.

Many attempts have been made to define the factors increasing the likelihood of sternotomy specifically, but a general consensus has still not been reached. Flati et al. [9], in 2005, defined the sternotomy approach "inevitable" in the presence of an iceberg-shaped retrotracheal goitre with $>70 \%$ of the mass lying in the mediastinum. Later, de Perrot et al. [7] in 2007, highlighted the need to perform a sternotomy in goitres $>10 \mathrm{~cm}$, in patients previously submitted to cervical thyroidectomy, and in the presence of invasive carcinoma or ectopic goitre. Burns et al. [1] performed a sternotomy in only 3/140 patients with retrotracheal goitre, since, in their opinion, the most significant factors giving rise to suspicion of the need to perform sternotomy are CT evidence of adherence to the surrounding mediastinal tissues and extension of the goitre to, or below, the aortic arch. More recently, Cohen [4] identified four factors significantly increasing the need to perform sternotomy: (a) the presence of malignancy, (b) involvement of the posterior mediastinum, (c) extension of the goitre below the aortic arch and (d) the presence of ectopic goitre. Cohen [4] suggests that the presence of symptoms is correlated more with the amount of growth of the thyroid at the level of the thoracic inlet, 
where the tracheae may be more easily compressed by the presence of the sternal manubrium, than by the total mass of the mediastinal thyroid.

Other authors have found that previous thyroid surgery could be a factor increasing the likelihood of sternotomy, due to the frequent finding of adhesions with surrounding tissues $[9,12]$. Preoperative estimation of thyroid volume, by means of CT scan, can be an effective predictor of which patients are likely to require a thoracic approach [9]. However, the final decision as to whether to perform sternotomy can be reached only intra-operatively, and the choice is related to the experience of the surgeon. In conclusion, even if retrosternal goitre thyroidectomy is performed by a skilled surgical team, familiar with its unique pitfalls, the assistance of a thoracic surgeon may be required.

\section{CONCLUSIONS}

This case indicates that diving goitre should be considered in the differential diagnosis of retrotracheal mediastinal masses. The preoperative diagnosis is essential and is primarily based on CT imaging findings. Sternotomy (partial or complete) or posterolateral mini-thoracotomy is required for the mass resection. Prognosis is excellent following a successful excision.

\section{REFERENCES}

1. Burns P, Doody J, Timon C. Sternotomy for substernal goitre: an otolaryngologist's perspective. J Laryngol Otol. 2008; 122(5): 495-499, doi:10.1017/ S0022215107000047, indexed in Pubmed: 17623491.

2. Chong $\mathrm{CF}$, Cheah WK, Sin FL, et al. Posterior mediastinal goiter. Asian Cardiovasc Thorac Ann. 2004; 12(3): 263-265, doi:10.1177/021849230401200319, indexed in Pubmed: 15353470.

3. Cohen J, Cho H. Surgery for substernal goiters. Oper Techn Otolaryngol Head Neck Surg. 1994; 5(2): 118-125, doi: 10.1016/1043-1810(94)90045-0.

4. Cohen J. Substernal goiters and sternotomy. Laryngoscope. 2009; 119(4): 683-688, doi: 10.1002/lary.20102.

5. Cougard P, Matet $P$, Goudet $P$, et al. Les goitres plongeants: 218 cas operes. Ann Endocrinol (Paris). 1992; 53: 230-235.
6. Dahan M, Gaillard J, Eschapasse H. Surgical treatment of goiters with intrathoracic development. In: Delarue M (ed) International trends in general thoracic surgery. Thoracic Surgery: Frontiers and uncommon neoplasms. Mosby, St Louis, Volume 5. 1989; 240: 246.

7. de Perrot M, Fadel E, Mercier O, et al. Surgical management of mediastinal goiters: when is a sternotomy required? Thorac Cardiovasc Surg. 2007; 55(1): 39-43, doi: 10.1055/ s-2006-924440, indexed in Pubmed: 17285472.

8. De Souza, Smith PE. Retrosternal goiter. J Otolaryngol. 1983; 12: 393-396.

9. Flati G, De Giacomo T, Porowska B, et al. Surgical management of substernal goitres. When is sternotomy inevitable? Clin Ter. 2005; 156(5): 191-195, indexed in Pubmed: 16382967.

10. Hashmi SM, Premachandra DJ, Bennett AMD, et al. Management of retrosternal goitres: results of early surgical intervention to prevent airway morbidity, and a review of the English literature. J Laryngol Otol. 2006; 120(8): 644-649, doi: 10.1017/S0022215106000995, indexed in Pubmed: 16884549.

11. Huins CT, Georgalas C, Mehrzad H, et al. A new classification system for retrosternal goitre based on a systematic review of its complications and management. Int J Surg. 2008; 6(1): 71-76, doi: 10.1016/j.ijsu.2007.02.003, indexed in Pubmed: 17416216.

12. Monchik JM, Materazzi G. The necessity for a thoracic approach in thyroid surgery. Arch Surg. 2000; 135(4): 467-71; discussion 471, indexed in Pubmed:10768714.

13. Sancho JJ, Kraimps JL, Sanchez-Blanco JM, et al. Increased mortality and morbidity associated with thyroidectomy for intrathoracic goiters reaching the carina tracheae. Arch Surg. 2006; 141(1): 82-85, doi: 10.1001/archsurg.141.1.82, indexed in Pubmed: 16415416.

14. Shahian DM, Rossi RL. Posterior mediastinal goiter. Chest. 1988; 94(3): 599-602, indexed in Pubmed: 3409742.

15. Spartalis ED, Karatzas T, Charalampoudis $P$, et al. Neglected papillary thyroid carcinoma seven years after initial diagnosis. Case Rep Oncol Med. 2013; 2013: 148973, doi: 10.1155/2013/148973, indexed in Pubmed: 23401818.

16. Vadasz P, Kotsis L. Surgical aspects of 175 mediastinal goiters. Eur J Cardiothorac Surg. 1998; 14(4): 393-397, indexed in Pubmed: 9845144.

17. White ML, Doherty GM, Gauger PG. Evidence-based surgical management of substernal goiter. World J Surg. 2008; 32(7): 1285-1300, doi:10.1007/s00268-008-9466-3, indexed in Pubmed: 18266028. 
